\title{
Solitary Dural Metastasis from Renal Cell Carcinoma Mimicking Meningioma Nine Years After Complete Remission: Case Report and A Literature Review
}

\section{Sidani $\mathrm{C}^{1}$, Rodriguez $\mathrm{JR}^{2 *}$ and Rahal $\mathrm{ON}^{3}$}

${ }^{1}$ Diagnostic Neuroradiology Program, Jackson Memorial Hospital, University of Miami, USA ${ }^{2}$ Department of Medicine, Jackson Memorial Hospital, University of Miami, Miami, Florida, USA

${ }^{3}$ Medical Student, Saba University School of Medicine, Netherland

\begin{abstract}
The clinical presentation of metastatic renal cell carcinoma mimicking an extra axial meningioma, almost a decade after radical nephrectomy is a rare occurrence. This study examined the diagnostic characteristics of a solitary metastatic lesion with the characteristics of an extra axial meningioma, the most common extra axial tumor in adults. A 67-year-old male presented with new neurologic symptoms, 9 years after right radical nephrectomy, without evidence of thoracic or abdominopelvic recurrence. Imaging, including CT, Octreotide scan, and 1.5 T conventional sequences demonstrated an extra axial enhancing mass with evidence of a Dural tail within the left cerebella medullary angle and cistern. These images were inconclusive in regards to truly differentiating the lesion. $3 \mathrm{~T}$ with SWI sequences and spectroscopy demonstrated hemorrhagic components of the lesion with associated superficial siderosis. The SWI images were vital in differentiation of the lesions. We present four additional cases of extra axial renal cell carcinomas mimicking meningioma radiographically.
\end{abstract}

Keywords: Extra axial brain lesions; Meningioma; Renal cell carcinoma

\section{Introduction}

Intracranial metastasis is a leading cause of morbidity and mortality and the most common neurologic complication diminishing quality of life in cancer patients [1]. With improvements in diagnostic and treatment modalities, the increase in survival of patients with primary extra cranial malignancies has been met with an increased incidence of metastases [2]. Intracranial metastases are the most common brain tumors in adults, affecting up to $30 \%$ of cancer patients, most of times presenting as an intra-axial lesion and very rarely as an extra axial mass [3]. Extra-axial tumors involve brain tissues other than the brain parenchyma; for example, choroid plexus, ventricles, and dura. Meningiomas are by far the most common extra axial tumors in adults and make up approximately $13 \%$ to $26 \%$ of all intracranial neoplasms [4]. Meningiomas classically present with a Dural tail, although this sign is not specific [1]. Extra-axial solitary lesions involving the dura with the classic features of meningiomas in patients with a primary malignancy provides a diagnostic dilemma with conventional CT and MRI modalities [1,3]. Multifocal meningiomas may mimic metastasis, while a single metastatic lesion, located in the expected location of a meningioma, may lead to incorrect diagnoses particularly in patients with no prior history of cancer, or in cases like ours, where cancer is presumed to have been cured [4]. Most meningioma mimickers involve primary breast and prostate carcinoma [5]. There have been four cases reported in the literature of misdiagnosed primary extra-axial meningiomas found to be renal cell carcinoma (RCC) pathologically $[3,6-8]$.

The radiological or clinical misdiagnosis of Dural metastasis for a meningioma can delay surgery and can cause harmful consequences to cancer patients [5]. In many cases pathological examination is necessary to make the correct diagnosis. In this report, we present the sixth case of a solitary RCC involving the dura, mimicking a meningioma and presenting a diagnostic dilemma in a patient with history of renal cell carcinoma that was disease free for nine years.

In addition, we demonstrate how "conventional sequences" can be confusing, and how the correct pre-operative diagnosis was accomplished with SWI sequences on a $3 \mathrm{~T}$ magnet. We also present a literature review of the four extranidal RCC metastases with characteristics of meningiomas.

\section{Clinical Presentation}

A 67-year-old man presented with episodic headaches, dysphagia, difficulty sleeping, nausea, vomiting, and weight loss. The patient has undergone a right flank radical nephrectomy 9 years earlier for resection of a renal cell carcinoma. CT brain demonstrated an extra axial hyperdense mass in the cerebellum medullary angle and cerebellum medullary cistern (Figure 1) with primary meningioma as the initial diagnosis, however metastatic disease was a consideration, given the patient's history. Octreoscan was performed at an outside facility and showed positive metabolic uptake at the level of the lesion (Figure 2). The result however was not conclusive as both meningioma and renal cell carcinoma metastasis (amongst other etiologies which were not a consideration in this case) have been described to show uptake with this modality scan [9]. MRI at $1.5 \mathrm{~T}$ magnet strength without and with intravenous contrast with conventional sequences demonstrated a well-defined Dural based solid enhancing lesion with a small Dural tail (Figure 3). The imaging features were suggestive of a meningioma, however given the patients history and new symptoms metastasis had to be excluded with more certainty.

A follow up 3.0 T MRI brain with spectroscopy was recommended, with the goal of assessing alanine peak, found to be elevated in meningiomas and not in metastasis disease [10]. During the acquisition, the lesion showed diffuse low signal on SWI, indicative of its hemorrhagic nature. Extensive susceptibility was also demonstrated in the subarachnoid spaces, particularly of the left posterior fossa, indicative of superficial siderosis (Figure 4). These findings were

*Corresponding author: Rodriguez JR, Department of Medicine, Jackson Memorial Hospital/University of Miami Hospital, Miami, Florida, USA, Tel: 305-968-9252; E-mail: jose.rodriguez2@jhsmiami.org

Received July 14, 2018; Accepted July 23, 2018; Published July 27, 2018

Citation: Sidani C, Rodriguez JR, Rahal ON (2018) Solitary Dural Metastasis from Renal Cell Carcinoma Mimicking Meningioma Nine Years After Complete Remission: Case Report and A Literature Review. J Clin Case Rep 8: 1144. doi: 10.4172/2165-7920.10001144

Copyright: $\odot 2018$ Sidani C, et al. This is an open-access article distributed under the terms of the Creative Commons Attribution License, which permits unrestricted use, distribution, and reproduction in any medium, provided the original author and source are credited. 
Citation: Sidani C, Rodriguez JR, Rahal ON (2018) Solitary Dural Metastasis from Renal Cell Carcinoma Mimicking Meningioma Nine Years After Complete Remission: Case Report and A Literature Review. J Clin Case Rep 8: 1144. doi: 10.4172/2165-7920.10001144

Page 2 of 3

\begin{tabular}{|c|c|c|c|c|}
\hline Author and year & Symptoms & Location & Age & Recurrence after primary (in years) \\
\hline Heary et al. 2014 & Upper thoracic pain and lower-extremity paresthesia & Intradural extra medullary & 57 & Undetermined (no primary RCC at time of resection) \\
\hline Present case & $\begin{array}{c}\text { Headache, dysphagia, difficulty sleeping, nausea \& vomiting, } \\
\text { weight loss }\end{array}$ & Intracranial extra axial & 67 & 9 \\
\hline Bademci et al. 2008 & Headache, speech disturbance, hemiparesis, confusion & Intracranial extra axial & 68 & 20 \\
\hline Montano et al. 2007 & Left faciobrachiocrural paresis & Intracranial extra axial & 65 & \\
\hline Laidlaw et al. 2004 & Right leg and hand weakness, unsteady gait, headaches & Intracranial extra axial & 74 & Undetermined (diagnosed at same time as primary) \\
\hline
\end{tabular}

Table 1: Renal cell carcinomas mimicking meningiomas.

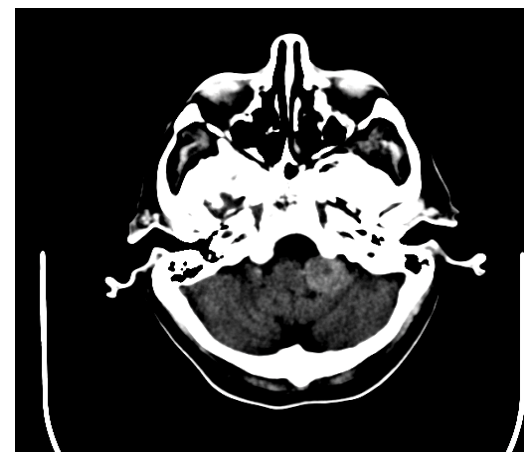

Figure 1: CT brain demonstrates a solid dense extra-axial lesion inferior to the $\mathrm{CP}$ angle, at the region of the left medulla cerebellum region/medulla cerebellum cistern.

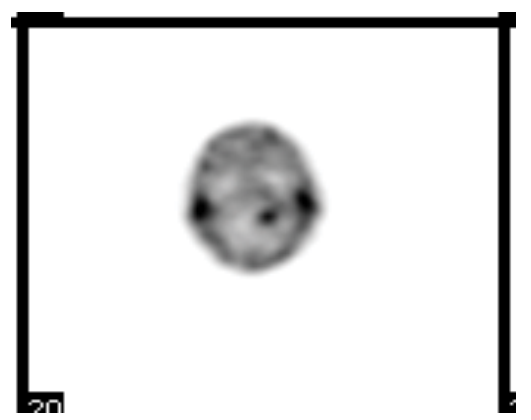

Figure 2: Octreotide scan demonstrate increased uptake at the left medulla cerebellum region.

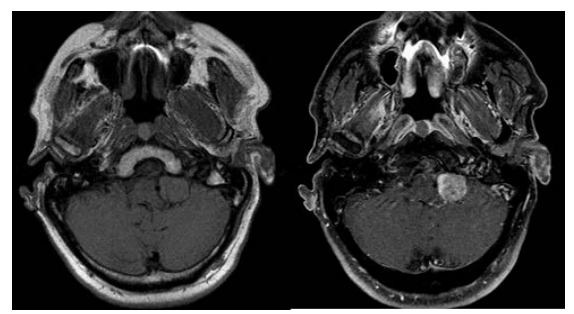

Figure 3: MRI at 1.5 T. Solid, isointense T1, enhancing extra-axial lesion, below the $\mathrm{CP}$ angle at the left medulla cerebellum level.

consistent with a hemorrhagic lesion and the diagnosis of renal cell metastasis was favored while meningioma was felt unlikely. The spectroscopy study was inconclusive due to the presence of hemorrhage in the lesion. Surgical intervention was recommended and ten days later the patient underwent a craniotomy, however there was noted hemorrhaging and the procedure was aborted. The lesion was endovascularly embolized with Onyx, followed by successful and uneventful re-resection. Pathology was consistent with RCC. The patient did well postoperatively (Table 1).

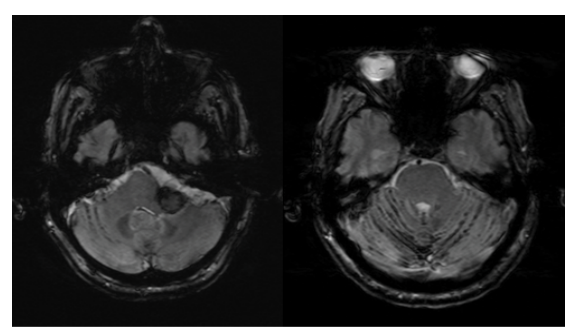

Figure 4: SWI performed on $3 \mathrm{~T}$ magnet demonstrates low signal in the lesion (indicating intra lesional hemorrhage) and low signal in the subarachnoid spaces of the posterior fossa indicating superficial siderosis. None of the findings were demonstrated on $\mathrm{CT}$ or MRI at $1.5 \mathrm{~T}$.

\section{Discussion}

Meningioma, the most common intracranial neoplasm in adults is clinically and radiologically mimicked by other extra axial lesions such as infectious/inflammatory diseases (Tuberculosis and Rheumatoid arthritis, respectively), hematopoietic neoplasms (lymphomas), and metastases [11]. Meningioma mimicking Dural metastases are rare however, with case studies primarily involving breast and prostate carcinomas [5]. Furthermore, it is well known that tumor-to-tumor metastasis can occur, with meningiomas being the most common intracranial recipients of lung and breast carcinomas [12,13]. Caroli et al. demonstrated in a case study of 63 published cases that meningiomas comprised $84 \%$ of the recipient tumors from extra-axial primaries. Multiple cases of RCC metastasizing to meningiomas have been described as part of the tumor to tumor phenomenon, but in these cases, to meet the tumor-to-tumor criteria the spread must be to a true independent original neoplasm [14]. In this literature review, we present six cases, including our report, of solitary metastatic renal cell carcinoma presenting as an extra axial meningioma.

Conventional MRI may not always reliably distinguish meningioma from other extra-axial lesions. Kremer et al. reported that MR Perfusion imaging might distinguish meningiomas from other extra axial lesions [15]. MR perfusion offers characteristics of a lesion at the capillary level and measures relative cerebral blood volume (rCBV) correlating with tumor vascularity where high rCBV reflects high rate of neo-angiogenesis [16]. Kremer et al. studied 22 extra-axial lesions (16 meningiomas and 6 Dural based meets) appearing similar on conventional MR imaging and concluded that $\mathrm{rCBV}$ is significantly lower for lung, breast, rectal carcinomas as well as lymphomas in comparison to the high rCBV of meningiomas. The study however did not include any cases of renal cell carcinoma.

Other diagnostic tools that may help delineate possible differences between metastatic disease and primary meningiomas is MR spectroscopy. Specifically, alanine peaks have been found to be elevated in meningiomas and not in high-grade gliomas or cerebral metastases [17]. Technically, however, MR spectroscopy may be limited when lesions are small, hemorrhagic, calcified, or close to the skull. 
Citation: Sidani C, Rodriguez JR, Rahal ON (2018) Solitary Dural Metastasis from Renal Cell Carcinoma Mimicking Meningioma Nine Years After Complete Remission: Case Report and A Literature Review. J Clin Case Rep 8: 1144. doi: 10.4172/2165-7920.10001144

As previously noted, Octreotide scans have been shown to be positive in both renal cell carcinomas and meningiomas. Renal cell carcinoma has a predilection to present with intra-tumoral hemorrhage, as seen in our case [18].

The utilization of early pathologic information for renal cell carcinomas may also serve as a starting point in the context of analyzing follow up imaging and perfusion studies. Chromosomal losses at $9 \mathrm{p}$ and $9 \mathrm{q}$ appeared to be associated with late brain metastasis from sporadic clear cell renal carcinoma [19].

In our case, the diagnosis was achieved by utilizing a higher strength magnet ( $3 \mathrm{~T}$ versus $1.5 \mathrm{~T}$ ) and the acquisition of SWI sequence which was able to demonstrate intralesional hemorrhage as well as chronic subarachnoid hemorrhage/superficial siderosis in the posterior fossa. These findings were not demonstrated on CT scan or MRI at $1.5 \mathrm{~T}$ and confirmed the malignant nature of the lesion.

\section{Conclusion}

Dural metastatic disease can mimic meningiomas and it might be difficult to distinguish the entities on conventional imaging resulting in delayed management (if metastasis is mistaken for meningioma) or in unnecessary surgery (if meningioma is mistaken for metastasis). Scans done with $3 \mathrm{~T}$ magnets utilizing SWI imaging may help provide further detail due to the intrinsic features of the lesion and aid in the diagnosis. In cases of renal cell carcinoma, Dural metastasis should be kept a consideration even several years after the patient is declared disease free.

\section{References}

1. Nayak, Lakshmi, Abrey LE, Iwamoto FM (2009) Intracranial Dural metastases. Cancer 9: 1947-1953.

2. Marosi, Christine, Preusser M (2017) Milestones of the last 10 years: CNS cancer. Memo Springer Vienna 10: 18-21.

3. Bademci G (2008) Extremely delayed renal cell carcinoma metastasis mimicking convexity meningioma. Neurocirugía 19: 562-564.

4. Engelhard $\mathrm{HH}$ (2001) Progress in the diagnosis and treatment of patients with meningiomas: Diagnostic imaging, preoperative embolization. Surg Neurol 55: 89-101.
5. Savage, Natasha M (2011) Dural-based metastatic carcinomas mimicking primary CNS neoplasia: Report of 7 cases emphasizing the role of timely surgery and accurate pathologic evaluation. Int J Clin Exper Pathol 4: 530

6. Heary, Robert F (2014) Metastatic renal cell carcinoma, with a radiographically occult primary tumor, presenting in the operative site of a thoracic meningioma: Long-term follow-up: Case report. J Neurosurg Spine 21: 628-633.

7. Montano, Nicola (2007) Extremely delayed falx metastasis from renal cell carcinoma. Neurol 68: 1541-1542.

8. Laidlaw, John D, Kumar A, Chan A (2004) Dural metastases mimicking meningioma: Case report and review of the literature. J Clin Neurosci 11: 780-783.

9. Schmidt M, Scheidhauer K, Voth E, Hildebrandt G, Klug N, et al. (1998) Somatostatin receptor imaging in intracranial tumors. Eur J Nucl Med 25: 675-686.

10. Sibtain NA, Howe FA, Saunders DE (2007) The clinical value of proton magnetic resonance spectroscopy in adult brain tumors. Clin Radiol 62: 109-119.

11. Zimny A, Ssiadek M (2011) Contribution of perfusion weighted magnetic resonance imaging in the differentiation of meningiomas and other extra-axial tumors: Case reports and literature review. J Neurooncol 103: 777-783.

12. Lee A, Wallace C, Rewcastle B, Sutherland G (1998) Metastases to meningioma. AJNR 19: 1120-1122.

13. Caroli E, Salvati M, Giangaspero F, Ferrante L, Santoro A (2017) Intra meningioma metastasis as first clinical manifestation of occult primary breast carcinoma: Neurosurgical review. US National Lib Med 29: 49-54.

14. Erdogan, Hakan, Aydin MV, Tasdemiroglu E (2014) Tumor-to-tumor metastasis of the central nervous system. Turkish Neurosurg 29: 303-308.

15. Kremer S (2004) Contribution of dynamic contrast MR imaging to the differentiation between Dural metastasis and meningioma. Neuroradiol 46 642-648.

16. Kremer S (2003) Dynamic contrast-enhanced MRI: Differentiating melanoma and renal carcinoma metastases from high-grade astrocytomas and other metastases. Neuroradiol 45: 44-49.

17. Kim, Hwy Y, Kim JW, Hyun-Tai C, Paek SH, et al. (2012) Brain metastasis from renal cell carcinoma. Progress in Neurological Surgery Current and Future Management of Brain Metastasis pp: 163-175.

18. Gutenberg A (2014) Predictive chromosomal clusters of synchronous and metachronous brain metastases in clear cell renal cell carcinoma: Cancer genetics. US National Lib Med p: 17.

19. Mishra, Atul, Thomas B, Kapilamoorthy TR (2017) Susceptibility weighted Imaging: A problem solving tool in differentiation of cerebellopontine angle schwannomas and meningiomas. Neuroradiol J 30: 253-258. 